\title{
Maternal Depressive Symptoms, Employment, and Social Support
}

\author{
Dwenda Gjerdingen, MD, MS, Patricia McGovern, PhD, MPH, Laura Attanasio, BA, \\ Pamela Jo Johnson, MPH, PhD, and Katy Backes Kozhimannil, PhD, MPA
}

Objective: The purpose of this study was to characterize the relationship between maternal depressive symptoms and employment and whether it is mediated by social support.

Methods: We used data from a nationally representative sample of 700 US women who gave birth in 2005 and completed 2 surveys in the Listening to Mothers series, the first in early 2006, an average of 7.3 months postpartum, and the second an average of 13.4 months postpartum. A dichotomous measure of depressive symptoms was calculated from the 2-item Patient Health Questionnaire, and women reported their employment status and levels of social support from partners and others. We modeled the association between maternal employment and depressive symptoms using multivariate logistic regression, including social support and other control variables.

Results: Maternal employment and high support from a nonpartner source were both independently associated with significantly lower odds of depressive symptoms (adjusted odds ratio [AOR], 0.35 and $P=.011$, and AOR, $0.40, P=.011$, respectively). These relationships remained significant after controlling for mothers' baseline mental and physical health, babies' health, and demographic characteristics (AOR, 0.326 and $P=.015$, and AOR, 0.267 and $P=.025$, respectively).

Conclusions: Maternal employment and strong social support, particularly nonpartner support, were independently associated with fewer depressive symptoms. Clinicians should encourage mothers of young children who are at risk for depression to consider ways to optimize their employment circumstances and "other" social support. (J Am Board Fam Med 2014;27:87-96.)

Keywords: Behavioral Sciences, Mental Health, Postpartum Care, Social Problems

Postpartum depression (PPD), self-reported by 11.7\% to $20.4 \%$ of women who give birth, ${ }^{1}$ is a serious complication of childbirth, with significant adverse

This article was externally peer reviewed.

Submitted 22 April 2013; revised 22 July 2013; accepted 5 August 2013.

From the Department of Family Medicine and Community Health, University of Minnesota Medical School, Minneapolis (DG); Medica Research Institute (PJJ) and Divisions of Environmental Health Sciences (PM), Health Policy and Management (LA, KBK), and Epidemiology and Community Health (PJJ), University of Minnesota School of Public Health, Minneapolis.

Funding: This study was supported by a Grant-in-Aid from the University of Minnesota Office of the Vice President and Vice Provost for Research and by the Building Interdisciplinary Research Careers in Women's Health Grant (K12HD055887), sponsored by the National Institutes of Health. This research also was supported by a grant from the Eunice Kennedy Shriver National Institute for Child Health and Human Development (R03HD070868).

Conflict of interest: none declared.

Corresponding author: Dwenda Gjerdingen, MD, MS, Department of Family Medicine and Community Health, University of Minnesota, 580 Rice St., St. Paul, MN 55103 (E-mail: dgjerdin@umphysicians.umn.edu). effects on both the mother and her infant. For the mother, PPD can produce mental and physical symptoms that adversely affect her quality of $\operatorname{life}^{2}$ and productivity $^{3}$; for the infant, PPD can disrupt motherinfant interactions, bonding, feeding, and sleep. ${ }^{4}$ PPD treatment traditionally has consisted of antidepressants and/or psychotherapy. ${ }^{5-8}$

Social support may play a critical role in both the development and treatment/resolution of PPD. Social support has long been considered important to mothers; this was highlighted in an analysis revealing that women's first major postpartum concern was the need for social support. ${ }^{9}$ The Agency for Health care Research and Quality reported that a low level of social support was a consistent risk factor for PPD. ${ }^{10}$ The relationship between social support and PPD is likely bidirectional; social support may be even more important for depressed mothers, given a newborn's significant needs and the potential impact of depression on a woman's ability to functionally meet those needs. 
Social support encompasses 4 primary dimensions: informational, material, emotional, and peer/ comparison (help given by someone in a similar situation). ${ }^{11}$ Prior research has consistently demonstrated a relationship between postpartum mental health and overall social support, ${ }^{12-20}$ emotional support from a partner, ${ }^{21-22}$ practical support from a partner, ${ }^{23}$ and various dimensions of social support from other (nonpartner) sources. ${ }^{24,25}$ These aspects of social support seem to coalesce to support postpartum mental health. For example, in a recent study of 168 London women who had recovered from PPD, specific types of support that were identified as essential to recovery included the partner's emotional and practical support, communication with partner, emotional support from friends, and prompt assessment by a health visitor. ${ }^{22}$ Furthermore, previous studies that looked at various sources of support found partner support (vs support by other relatives, friends, etc.) to be a stronger or more pervasive predictor of postpartum mental well-being. ${ }^{26-28}$

The contribution of social support to maternal mental health may be particularly important for mothers who return to formal employment after giving birth. Using US Census data on first-time mothers, Laughlin ${ }^{29}$ reports patterns of employment and childbirth between 2006 and 2008. Women are more likely to work outside the home both before and during their pregnancy than they were 30 to 40 years ago, and most (55\%) are employed into the last month of their pregnancy. Factors associated with maternal employment during pregnancy include older age ( $\geq 22$ years old vs younger), race and ethnicity (employment rates: non-Hispanic white, $75 \%$; Asian, $61 \%$; black, $52 \%$; and Hispanic, 42\%), and higher educational achievement (employment rates: $87 \%$ if more than a bachelor's degree, $23 \%$ if less than a bachelor's degree). In addition, more women are now returning to formal employment within a year of giving birth: $64 \%$ in 2005 to 2007 compared with $39 \%$ in 1976 to 1980 and $17 \%$ in 1961 to 1965 . Regardless of first-time mothers' pregnancy employment status, $44 \%$ are employed 3 months postpartum, 57\% by 6 months, and $64 \%$ by 12 months. ${ }^{29}$

There is currently little information on the relationship between employment after childbirth and postpartum mental health. Paid work theoretically might either enhance or detract from a woman's support systems and mental health, de- pending on the characteristics of her job. For example, in a Minnesota study of 817 employed mothers of infants, worse depression scores in the first 6 months after childbirth were associated with higher total workload (paid and unpaid work), lower job flexibility, lower social support, and having an infant with sleep problems. ${ }^{30}$ In addition, higher workloads and having an infant with sleep problems or fussy behavior were risk factors for poor postpartum mental health, whereas social support and increased perceived control over work and home activities provided a protective effect. ${ }^{12}$ Analyzing the same data set and controlling for available social support from family, friends, and the workplace, Grice and colleagues ${ }^{31}$ found that job spillover to home (eg, bringing work home) and home spillover to job (eg, taking telephone calls at work related to infant care) were each associated with poorer mental health.

Therefore, although there is strong evidence that social support is associated with better postpartum mental health, there is limited available research that directly examines the relationship between postpartum employment and social support and the combined interaction between mothers' mental health, employment, and social support. The purpose of this study was to characterize the relationship between maternal depressive symptoms and employment and whether this relationship is mediated by social support.

\section{Materials and Methods \\ General Procedures and Study Sample}

Data for this analysis come from 2 surveys in the Listening to Mothers (LTM) series: LTM II and its postpartum follow-up survey. The questionnaires and reports for the baseline and follow-up surveys are available at http://www.childbirthconnection. org/article.asp?ck $=10413$.

LTM II is a survey that was conducted in January and February 2006 among a nationally representative sample of 18- to 45-year-old women who gave birth to a single baby in a US hospital in 2005 . The LTM II baseline survey was completed an average (weighted mean) of 7.3 months after delivery (standard error, 0.15 months; range, 0.8-12.2 months); the follow-up survey was completed 6 months later, an average of 13.4 months postpartum (standard error, 0.15 months; range, 6.8-18.5 months). Our final weighted sample included 253 
employed and 447 unemployed women, for a total of 700 women. Of the 253 employed women, 173 (68.4\%) worked full-time and 80 (31.6\%) worked part-time.

The LTM II baseline survey contained information on childbirth and postpartum return to work, and the follow-up survey contained details on depressive symptoms, social support, and additional employment information. While respondents completed postpartum surveys at 2 separate times (the LTM II baseline survey and the LTM II follow-up survey 6 months later), our primary exposure and outcome variables both were measured in the follow-up survey.

Strictly speaking, the term postpartum generally refers to a period of weeks or a few months following delivery. However, in reality, many health-related effects of childbirth, including both physical and mental disorders, may persist for several months or longer. ${ }^{32,33}$ Therefore, consistent with the LTM II purposes to evaluate short- and longerterm childbirth-related experiences and outcomes, we conceptualize the follow-up interval at 13 months after delivery to be part of participants' postpartum experience.

\section{Variable Measurement}

\section{Employment Status}

Our primary comparison groups of interest were classified by postpartum employment status, which was based on self-report. Women who reported working full-time or part-time for an external employer at the time of the follow-up survey were coded as employed, and women who reported not working for an external employer during this time were coded as not employed. (We do not know how many unemployed women chose not to return to work or no longer had a job waiting for them.) Because of the timing of the depressive measures with respect to work, the following women were excluded from the analysis: those who returned to work before 8 weeks postpartum $(n=129)$, those who worked after childbirth but were self-employed ( $n=47)$, and those who were on extended maternity leave at the time of the follow-up survey $(\mathrm{n}=25)$.

\section{Social Support}

Social support was classified by type and source. The postpartum follow-up survey asked women about support available from married or unmarried partners and others (specific sources of other support were not elicited). For each source of support there were questions about how often 4 domains of support were available to the respondent. The 4 support domains were emotional (ie, listening to your concerns and giving good advice), practical (ie, helping you get things done or get needed information), affectionate (ie, showing you affection and helping you feel wanted), and enjoyment (ie, having fun or relaxing together). Response choices for support availability were none of the time (0), a little of the time (1), some of the time (2), most of the time (3), or all the time (4).

For each source of support (partner or other) we created an index by summing the responses for each domain of partner support and each domain of other support. Each index ranged from 0 to 16 . We divided the index into quartiles to indicate low, medium, high, or very high support from each potential source. We then created a dichotomous measure of high support or not from each source using a score of 8 as the cutpoint. Finally, we created a 4-category social support variable representing mutually exclusive categories of whether the woman reported an absence of high support from either source, high support from only a partner, high support from only another (nonpartner) source, or high support from both sources.

\section{Depression}

Our key outcome was a measure of depressive symptoms experienced by the respondent in the 2 weeks preceding completion of the follow-up survey. This was assessed using the 2-item Patient Health Questionnaire (PHQ-2). Women were asked how often over the past 2 weeks they had been bothered by little interest or pleasure in doing things and how often they had felt down, depressed, or hopeless. Response choices were "not at all," "several days," "more than half the days," or "nearly every day"; each response was scored 0 to 3, and the 2 responses were summed. A score of $\geq 3$ was coded as depressive symptoms. In a previous study of 6000 patients from 8 primary care clinics and 7 obstetrics-gynecology clinics, a PHQ-2 score of $>3$ had a sensitivity of $83 \%$ and specificity of $92 \%$ for identifying major depression when compared with a structured interview with a mental health professional. ${ }^{34}$ 


\section{Other Variables}

Additional control variables included race/ethnicity; age; marital status at the time of the postpartum survey; maternal education (less than high school to high school diploma, more than high school through college degree, more than college); 2005 household income (less than $\$ 50,000, \$ 50-\$ 100,000,>\$ 100,000$ ); health status of the child; whether this child was the respondent's first baby (parity); number of mother's major and minor physical problems in the first 8 weeks postpartum (excluding those that could be symptoms of depression); and whether the mother reported feelings of depression as a new minor or new major problem during the first 8 weeks postpartum. All these measures were based on respondent self-report.

\section{Analysis}

Analysis took place in 2 phases and made use of both descriptive statistics and multiple logistic regression modeling. In the first phase we used means and percentages to characterize the sample by postpartum employment status. We then summarized mean level of social support availability by postpartum employment status. For each available domain (emotional, practical, affection, enjoyment) and source (partner or other) of social support, we calculated the mean level of support by employment status and used adjusted Wald tests to determine whether support levels were significantly different for women who were employed postpartum compared with those who were not. We also examined the distribution of women across categories of the support index variables and tested whether these distributions differed by employment status.

In the second phase, which was a cross-sectional, multivariate analysis, we estimated the odds of depressive symptoms by employment status and social support using logistic regression and adjusting for all the covariates described above. In the first model we investigated the association of high partner support with depressive symptoms among partnered women. In a second model we examined the association of high "other" support with depressive symptoms among all participants. In a final set of sequentially built models, we included both partner and other support in the same model by using the 4-category social support variable. First, we estimated the unadjusted odds of postpartum depres- sive symptoms by employment status. Next we adjusted for social support. In the final fully adjusted model we estimated the odds of maternal depressive symptoms based on employment status and adjusting for social support as well as a full array of covariates. Women without a partner $(n=33)$ were excluded from all analyses that used the partner support variables. We conducted a sensitivity analysis that additionally controlled for time since delivery.

We also conducted a mediation analysis to determine whether any relationship between maternal employment and depressive symptoms was mediated by social support. First, we estimated the relationship between postpartum employment (independent variable) and depressive symptoms (dependent variable), not including social support but controlling for other potential confounders, which yielded an odds ratio (OR) of $0.36(P=.016)$. Second, we estimated the relationship between employment (independent variable) and social support (dependent variable) because a statistical relationship here would be required for social support to be a mediator of the relationship between prenatal employment and depressive symptoms. We estimated this effect using different specifications of the social support variable, including separately assessing high partner support and high other support. If no significant employment-social support relationship was observed, no further mediation analyses would be required.

All analyses were conducted using Stata statistical software version 12 (StataCorp LP, College Station, TX) using techniques for survey data. The survey analysis techniques accounted for the unequal probability of selection using sampling weights provided with data from the LTM II postpartum survey. Sampling weights adjusted the data to be nationally representative of women ages 18 to 45 who gave birth to a singleton baby in a US hospital in 2005. The weighting strategy used for the LTM II resulted in nationally representative distributions, ${ }^{35}$ but it does not inflate the sample to produce nationally representative weighted estimates of the population size.

\section{Results}

Descriptive statistics for the sample by employment status are presented in Table 1. Factors associated with maternal employment included being black and non-Hispanic, achieving higher levels of edu- 
Table 1. Sample Characteristics by Postpartum Employment Status

\begin{tabular}{|c|c|c|c|c|}
\hline Characteristics & $\begin{array}{c}\text { Not Employed } \\
(\mathrm{n}=447 ; 63.9 \%)\end{array}$ & $\begin{array}{c}\text { Employed } \\
(\mathrm{n}=253 ; 36.1 \%)\end{array}$ & $\begin{array}{c}\text { Total } \\
(\mathrm{n}=700)\end{array}$ & $P$ Value* \\
\hline \multicolumn{5}{|l|}{ Race/ethnicity $^{\dagger}$} \\
\hline Non-Hispanic white & 68.4 & 67.1 & 67.9 & \\
\hline Non-Hispanic black & 7.5 & 18.0 & 11.3 & \\
\hline Hispanic & 20.3 & 10.0 & 16.5 & \\
\hline Other race & 3.9 & 4.9 & 4.2 & .007 \\
\hline \multicolumn{5}{|l|}{ Maternal age $\left(\right.$ years) ${ }^{\dagger}$} \\
\hline $18-24$ & 27.7 & 31.8 & 29.2 & \\
\hline $25-29$ & 29.0 & 23.9 & 27.1 & \\
\hline $30-34$ & 25.3 & 30.0 & 27.0 & \\
\hline$\geq 35$ & 18.0 & 14.4 & 16.7 & .475 \\
\hline \multicolumn{5}{|l|}{ Educational attainment $^{\dagger}$} \\
\hline High school or less & 48.9 & 30.7 & 42.3 & \\
\hline College & 44.3 & 55.9 & 48.5 & \\
\hline More than college & 6.8 & 13.5 & 9.2 & .004 \\
\hline \multicolumn{5}{|l|}{ Marital status $^{\ddagger}$} \\
\hline Unmarried & 20.1 & 33.6 & 25.0 & \\
\hline Married & 79.9 & 66.4 & 75.0 & .013 \\
\hline \multicolumn{5}{|l|}{ Partnership status ${ }^{\ddagger}$} \\
\hline Does not have partner & 5.9 & 9.1 & 7.0 & \\
\hline Has partner & 94.1 & 90.9 & 93.0 & .369 \\
\hline \multicolumn{5}{|l|}{ Family income $^{\dagger}$} \\
\hline Low $(<\$ 50,000)$ & 54.7 & 43.5 & 50.6 & \\
\hline Medium $(\$ 50,000-99,999)$ & 32.3 & 36.0 & 33.6 & \\
\hline High $(\geq \$ 100,000)$ & 13.1 & 20.5 & 15.8 & .098 \\
\hline \multicolumn{5}{|l|}{ Baby's health status $^{\ddagger}$} \\
\hline Good health & 96.9 & 96.0 & 96.6 & \\
\hline Poor health & 3.1 & 4.0 & 3.4 & .558 \\
\hline \multicolumn{5}{|l|}{ Parity $^{\dagger}$} \\
\hline 1 & 33.8 & 47.8 & 38.9 & \\
\hline$>1$ & 66.2 & 52.2 & 61.2 & .016 \\
\hline \multicolumn{5}{|c|}{ Recent maternal depression (first 8 weeks) ${ }^{\ddagger}$} \\
\hline No depression & 63.5 & 61.5 & 62.8 & \\
\hline Minor depression & 24.4 & 23.4 & 24.0 & \\
\hline Major depression & 12.1 & 15.1 & 13.2 & .756 \\
\hline \multicolumn{5}{|c|}{ Maternal postpartum problems (mean) ${ }^{\S}$} \\
\hline Minor physical problems & 2.8 & 2.7 & 2.8 & .761 \\
\hline Major physical problems & 1.3 & 1.7 & 1.5 & .123 \\
\hline \multicolumn{5}{|c|}{ Depressive symptoms (PHQ-2 $=3+)^{\ddagger}$} \\
\hline No & 79.2 & 87.3 & 82.1 & \\
\hline Yes & 20.9 & 12.7 & 17.9 & .093 \\
\hline
\end{tabular}

Data are percentages unless otherwise indicated. Note that estimates are self-weighted in accordance with the survey design for national representativeness. Reported

${ }^{*} P$ values are from $\chi^{2}$ tests for categorical variables and adjusted Wald tests for continuous variables.

${ }^{\dagger}$ Measure was created from item(s) on the Listening to Mothers II survey (baseline).

${ }^{\ddagger}$ Measure was created from item(s) on the Listening to Mothers II-Postpartum survey (follow-up).

${ }^{\S}$ Measure was created from Listening to Mothers II baseline and postpartum surveys combined.

cation, and being a first time mother. Factors associated with not being employed included being Hispanic and married.
Table 2 reports the results for the distribution of social support by postpartum employment status. None of the social support measures differed sig- 
Table 2. Social Support: Component Measures and Social Support Scales by Postpartum Employment Status $(\mathrm{n}=700)$

\begin{tabular}{|c|c|c|c|c|}
\hline Measures/Scales & Not Employed & Employed & Total & $P$ value* \\
\hline \multicolumn{5}{|c|}{ Partner support (among women with partners) } \\
\hline \multicolumn{5}{|c|}{ Components of social support from partner/husband (mean) } \\
\hline Mean emotional support & 2.6 & 2.6 & 2.6 & .652 \\
\hline Mean practical support & 2.3 & 2.5 & 2.4 & .434 \\
\hline Mean affectionate support & 2.7 & 2.8 & 2.7 & .782 \\
\hline Mean enjoyment support & 2.4 & 2.5 & 2.4 & .652 \\
\hline \multicolumn{5}{|l|}{ Partner support index (\%) } \\
\hline Low $(0-4)$ & 15.9 & 10.6 & 14.0 & \\
\hline Medium (5-8) & 19.1 & 19.9 & 19.4 & \\
\hline High $(9-12)$ & 33.8 & 39.7 & 35.9 & \\
\hline Very high (13-16) & 31.3 & 29.9 & 30.8 & .565 \\
\hline \multicolumn{5}{|l|}{ Other support (among all women) } \\
\hline \multicolumn{5}{|c|}{ Components of social support from others (mean) } \\
\hline Mean emotional support & 2.5 & 2.4 & 2.5 & .242 \\
\hline Mean practical support & 2.0 & 2.2 & 2.1 & .199 \\
\hline Mean affectionate support & 2.1 & 2.0 & 2.1 & .347 \\
\hline Mean enjoyment support & 2.0 & 2.2 & 2.1 & .336 \\
\hline \multicolumn{5}{|l|}{ Other support index (\%) } \\
\hline Low $(0-4)$ & 15.1 & 18.6 & 16.4 & \\
\hline Medium (5-8) & 35.0 & 33.4 & 34.4 & \\
\hline High (9-12) & 35.8 & 34.0 & 35.1 & \\
\hline Very high (13-16) & 14.1 & 14.1 & 14.1 & .873 \\
\hline \multicolumn{5}{|c|}{$\begin{array}{l}\text { Combined support (among women who answered both } \\
\text { support questions) (\%) }\end{array}$} \\
\hline No high support & 23.0 & 20.7 & 22.2 & \\
\hline High partner support only & 27.1 & 29.0 & 27.8 & \\
\hline High other support only & 11.9 & 9.8 & 11.2 & \\
\hline High partner and other support & 38.0 & 40.6 & 38.9 & .886 \\
\hline
\end{tabular}

Women who do not have partners are excluded from calculations related to partner support variables, resulting in a weighted $\mathrm{n}$ of 651 for those variables.

* Reported $P$ values are from $\chi^{2}$ tests for categorical variables and adjusted Wald tests for continuous variables.

nificantly by employment status. The means for each component of social support ranged from 2.1 to 2.7 (scale range, $0-4$ ). Among women with partners, a majority had high $(35.9 \%)$ or very high $(30.8 \%)$ levels of partner support. Support levels from others were slightly lower, with $35.1 \%$ having high support and only $14.1 \%$ having very high support from sources other than a partner. More than a fifth of women $(22.2 \%)$ did not have a high level of support from either source. Nearly $40 \%$ had high support from both partners and others. Partner support was associated with other support (correlation coefficient: 0.270 for dichotomized support $[P<.001]$ and 0.318 for support index $[P<.001])$ among respondents in our study population.
The results for the multivariate logistic regression models including high partner or high other support are presented in Table 3. In both models, maternal employment was associated with significantly lower odds of depressive symptoms (adjusted OR [AOR], 0.35; $P=.011)$. Among women who had partners, high partner support was not associated with significantly lower odds of depressive symptoms after controlling for return to work and the other covariates (AOR, $0.59 ; P=.154$ ). In the model examining support from others, high social support was associated with significantly lower odds of depression (AOR, 0.40; $P=.011$ ).

Table 4 shows the results for the final series of regression models. In the unadjusted model, employment is associated with reduced odds of de- 
Table 3. Odds of Depressive Symptoms by Postpartum Employment Status and Social Support

\begin{tabular}{|c|c|c|c|c|c|c|}
\hline & \multicolumn{3}{|c|}{ Women with Partners $(\mathrm{n}=651)$} & \multicolumn{3}{|c|}{ All Women $(\mathrm{n}=700)$} \\
\hline & AOR & $95 \% \mathrm{CI}$ & $P$ & AOR & $95 \% \mathrm{CI}$ & $P$ \\
\hline \multicolumn{7}{|l|}{ Postpartum employment } \\
\hline Not employed & 1.00 & & & 1.00 & & \\
\hline Employed & 0.35 & $0.14-0.87$ & .023 & 0.35 & $0.16-0.79$ & .011 \\
\hline \multicolumn{7}{|l|}{ Social support } \\
\hline No high partner support & 1.00 & & & & & \\
\hline High partner support & 0.59 & $0.29-1.22$ & .154 & & & \\
\hline No high other support & & & & 1.00 & & \\
\hline High other support & & & & 0.40 & $0.20-0.81$ & .011 \\
\hline
\end{tabular}

Models control for race/ethnicity, age, education, marital status, family income, baby's health status, parity, maternal depression in the first 8 weeks postpartum, and a count of postpartum physical symptoms. Models are weighted to be nationally representative.

AOR, adjusted odds ratio; CI, confidence interval.

pressive symptoms, but this association is only marginally statistically significant (AOR, 0.51; $P=$ .090). After adjusting for high support from either a partner or another source, the relationship remains similar (AOR, 0.51; $P=.098$ ). In the fully adjusted model employment is associated with significantly lower odds of depressive symptoms (AOR, 0.33; $P=.015$ ). In addition, in the fully adjusted model women with high levels of support from both a partner and another source or high support from other (nonpartner) sources had lower odds of depressive symptoms compared with women who did not report high support from either source. Having high partner support only was not significantly associated with depressive symptoms compared with lacking high support from either source. The sensitivity analysis did not alter these results.
The mediation analysis yielded an OR of 0.36 $(P=.016)$ for the association of employment with depressive symptoms. However, there was no significant relationship between employment and social support when controlling for other covariates, so we concluded that social support was not a mediator of the relationship between postpartum return to work and depressive symptoms.

\section{Discussion}

Our results showed that postpartum employment and social support were independently associated with fewer depressive symptoms, even after controlling for demographic and health characteristics. These findings are consistent with previous research showing a positive association between post-

Table 4. Odds of Depressive Symptoms by Postpartum Employment Status and Combined Forms of Social Support $(n=651)$

\begin{tabular}{|c|c|c|c|c|c|c|c|c|c|}
\hline & \multicolumn{3}{|c|}{ Unadjusted Model } & \multicolumn{3}{|c|}{$\begin{array}{l}\text { Model Adjusted } \\
\text { for Social Support }\end{array}$} & \multicolumn{3}{|c|}{$\begin{array}{l}\text { Fully Adjusted } \\
\text { Model* }^{*}\end{array}$} \\
\hline & OR & $95 \% \mathrm{CI}$ & $\mathrm{P}$ & OR & $95 \% \mathrm{CI}$ & $\mathrm{P}$ & OR & $95 \%$ CI & $\mathrm{P}$ \\
\hline \multicolumn{10}{|l|}{ Postpartum employment } \\
\hline Not employed & 1.00 & & & 1.00 & & & 1.00 & & \\
\hline Employed & 0.51 & $0.24-1.11$ & .090 & 0.51 & $0.23-1.13$ & .098 & 0.33 & $0.13-0.81$ & .015 \\
\hline \multicolumn{10}{|l|}{ Social support } \\
\hline No high support & & & & 1.00 & & & 1.00 & & \\
\hline High partner support only & & & & 0.40 & $0.18-0.92$ & .031 & 0.51 & $0.21-1.26$ & .145 \\
\hline High other support only & & & & 0.21 & $0.07-0.67$ & .009 & 0.27 & $0.08-0.85$ & .025 \\
\hline High partner and other support & & & & 0.31 & $0.14-0.72$ & .006 & 0.37 & $0.15-0.91$ & .031 \\
\hline
\end{tabular}

Models are weighted to be nationally representative. Women without partners are excluded.

*The fully adjusted model controls for race/ethnicity, age, education, marital status, family income, baby's health status, parity, maternal depression in the first 8 weeks postpartum, and a count of postpartum physical symptoms.

$\mathrm{OR}$, odds ratio; CI, confidence interval. 
partum mental health and social support ${ }^{10}$ and between postpartum depressive symptoms and unemployment. ${ }^{36-38}$ Our findings differ, however, from previous research ${ }^{26-28}$ in that "other support" (vs partner support) seemed to be a stronger predictor of mothers' mental health. While reasons for this difference are not known, it could be related to differences in sample or support measures between studies. Nevertheless, this finding suggests an important role for social support provided by other family members and friends.

One might ask whether the relationship between good mental health and employment is simply due to the "healthy worker effect," whereby individuals' poor physical health may contribute to both unemployment and depressive symptoms. ${ }^{39}$ The results of our multivariate regression model sequence (Table 4) suggest that the healthy worker effect does not completely explain the inverse relationship between employment and depressive symptoms because this relationship remained significant even after adjusting for baseline maternal mental and physical health and demographic characteristics.

We also considered whether the social support framework found in many places of employment (eg, maternity-related employer accommodations, interpersonal relationships with colleagues) might explain the better mental health of women who returned to work. Our fully adjusted analyses indicated that, even when we controlled for social support from different sources (partner or other, which could include work-related support), employment continued to be independently associated with fewer depressive symptoms.

Social psychologist Marie Jahoda, ${ }^{40}$ well-known for her theory of ideal mental health, identified 5 employment by-products that are vital to one's well-being: time structure, social contacts and shared experiences, social purposes, status and identity, and regular activity. She stressed that even when one or more of these by-products is unpleasant (eg, too rigid or demanding a time structure), unpleasant ties to reality are preferable to their absence. This theory argues that social support may be one important reason for the positive employment-mental health connection but that there are likely other nonmonetary benefits of employment, such as structure, purpose, shared experiences, and self-identity (all unmeasured here), that contribute to the inherent value of employment, even in the lives of busy mothers. Further research might help to clarify the importance of such work characteristics.

Our analysis has implications for pregnant and childbearing women as well as their clinicians. These results provide evidence that social support may help to prevent or ameliorate depressive symptoms in mothers of young children. Support from a source other than a partner seems to play a particularly important role; such support could originate in the workplace or elsewhere. Women should identify the specific types of support they may need and seek to secure this support. Clinicians should discuss employment circumstances as well as social support with pregnant women both during and after pregnancy; such discussions may be particularly beneficial for women at risk for depression.

\section{Strengths and Limitations}

Strengths of this study included the use of a nationally representative sample of US childbearing women, with rich data allowing for the examination of the issues of employment, social support, and depressive symptoms while also controlling for key social, demographic, and health variables. Since survey questions addressed postpartum problems during 2 time periods, we were able to use information on depressive symptoms in the early postpartum period (first 8 weeks) to isolate the new onset of depressive symptoms contemporaneous with employment status at the time of the follow-up survey (at 13 months, on average).

Despite these strengths, there are limitations that affect the interpretation of our results. The LTM surveys did not collect information on occupational characteristics or employment-related stress, which may have aided interpretation but would be unlikely to alter our main findings. Measurement of depressive symptoms was limited to a 2-question screen (PHQ-2), which has been used with success in prior research. Regarding social support, domains assessed by these data are not fully consistent with prior research, and data on nonpartner sources of social support were not explicitly ascertained. These additional details would have enhanced our interpretation but would not likely appreciably affect results. Because of the contemporaneous occurrence of several key variables, we were not able to determine the directionality of the relationships we observed between postpartum employment, social support, and depressive symptoms. Although the LTM study was designed as a 
longitudinal study, most of the variables included in the follow-up survey were not present in the baseline survey; as a consequence, the analyses presented here focused on follow-up survey variables, making this primarily a cross-sectional study. Furthermore, the retrospective nature of the follow-up survey may have resulted in recall bias. Finally, the exclusion of women who were self-employed or returned to work before 8 weeks postpartum may have produced selection bias, and sample size limited our ability to explore subgroups of interest.

\section{Conclusion}

Employment was consistently associated with lower odds of depressive symptoms at 13 months after delivery, a relationship that may be explained by a combination of factors, including the healthy worker effect and nonmonetary employment benefits. It is important that this positive work-mental health relationship persisted after controlling for social support, physical problems, and other demographic variables. Consistent with prior literature, social support, particularly support from a source other than the woman's partner, also was associated with lower odds of maternal depressive symptoms, regardless of employment status. New mothers may benefit from identifying needed support and sources of support to ameliorate depressive symptoms during a time of heightened vulnerability.

\section{References}

1. Centers for Disease control and Prevention (CDC). Prevalence of self-reported postpartum depressive symptoms -17 states, 2004-2005. MMWR Morb Mortal Wkly Rep 2008;57:361-6.

2. Da Costa D, Dritsa M, Rippen N, Lowensteyn I, Khalife S. Health-related quality of life in postpartum depressed women. Arch Womens Ment Health 2006;9:95-102.

3. Stewart WF, Ricci JA, Chee E, Hahn SR, Morganstein D. Cost of lost productive work time among US workers with depression. JAMA 2003; 289:3135-44.

4. Field T. Postpartum depression effects on early interactions, parenting, and safety practices: a review. Infant Behav Dev 2010;33:1-6.

5. Appleby L, Warner R, Whitton A, Faragher B. A controlled study of fluoxetine and cognitive-behavioural counselling in the treatment of postnatal depression. Br Med J 1997;314:932-6.

6. Dennis CL, Hodnett ED. Psychosocial and psychological interventions for treating postpartum depression. Cochrane Database Syst Rev 2007;(4): CD006116.

7. Misri S, Reebye P, Corral M, Milis L. The use of paroxetine and cognitive-behavioral therapy in postpartum depression and anxiety: a randomized controlled trial. J Clin Psychiatry 2004;65:1236-41.

8. Yonkers KA, Lin H, Howell HB, Heath AC, Cohen LS. Pharmacologic treatment of postpartum women with new-onset major depressive disorder: a randomized controlled trial with paroxetine. J Clin Psychiatry 2008;69:659-65.

9. Kanotra S, D'Angelo D, Phares TM, Morrow B, Barfield WD, Lansky A. Challenges faced by new mothers in the early postpartum period: an analysis of comment data from the 2000 Pregnancy Risk Assessment Monitoring System (PRAMS) survey. Matern Child Health J 2007;11:549-58.

10. Agency for HealthCare Research and Quality. Effective Health Care Program: efficacy and safety of screening for postpartum depression, March, 92012. Available from: http://www.effectivehealthcare.ahrq.gov/ search-for-guides-reviews-and-reports/?pageaction = displayproduct\&productID=997. Accessed March 9, 2012.

11. Logsdon MC, Usui W, Birkimer JC, McBride AB. The Postpartum Support Questionnaire: reliability and validity. J Nurs Meas 1996;4:129-42.

12. McGovern P, Dagher RK, Rice HR, et al. A longitudinal analysis of total workload and women's health after childbirth. J Occup Environ Med 2011; 53:497-505.

13. Howell EA, Mora PA, DiBonaventura MD, Leventhal H. Modifiable factors associated with changes in postpartum depressive symptoms. Arch Womens Ment Health 2009;12:113-20.

14. Panthangi V, West P, Savoy-Moore RT, Geeta M, Reickert E. Is seasonal variation another risk factor for postpartum depression? J Am Board Fam Med 2009;22:492-7.

15. Xie RH, He G, Koszycki D, Walker M, Wen SW. Prenatal social support, postnatal social support, and postpartum depression. Ann Epidemiol 2009;19: 637-43.

16. Surkan PJ, Peterson KE, Hughes MD, Gottlieb BR. The role of social networks and support in postpartum women's depression: a multiethnic urban sample. Matern Child Health J 2006;10:375-83.

17. Robertson E, Grace S, Wallington T, Stewart DE. Antenatal risk factors for postpartum depression: a synthesis of recent literature. Gen Hosp Psychiatry 2004;26:289-95.

18. Beck CT. Predictors of postpartum depression. Nurs Res 2001;50:275-85.

19. O'Hara MW, Swain AM. Rates and risk of postpartum depression-a meta-analysis. Int Rev Psychiat 1996;8:37-54.

20. Webster J, Nicholas C, Velacott C, Cridland N, Fawcett L. Quality of life and depression following 
childbirth: impact of social support. Midwifery 2011; 27:745-9.

21. Bielinski-Blattmann D, Lemola S, Jaussi C, Stadlmayr W, Grob A. Postpartum depressive symptoms in the first 17 months after childbirth: the impact of an emotionally supportive partnership. Int J Public Health 2009;54:333-9.

22. Di Mascio V, Kent A, Fiander M, Lawrence J. Recovery from postnatal depression: a consumer's perspective. Arch Womens Ment Health 2008;11: 253-7.

23. Sayil M, Güre A, Uçanok U. First time mothers' anxiety and depressive symptoms across the transition to motherhood: associations with maternal and environmental characteristics. Womens Health 2006;44:61-77.

24. Xie RH, Yang J, Liao S, Xie H, Walker M, Wen SW. Prenatal family support, postnatal family support and postpartum depression. Aust N Z J Obstet Gynaecol 2010;50:340-5.

25. Dennis CL, Hodnett E, Reisman HM, et al. Effect of peer support on prevention of postnatal depression among high risk women: multisite randomized controlled trial. BMJ 2009;338:a3064.

26. Gjerdingen D, Chaloner KM. The relationship of women's postpartum mental health to employment, childbirth, and social support. J Fam Pract 1994;38: 465-72.

27. Hopkins J, Campbell SB. Development and validation of a scale to assess social support in the postpartum period. Arch Womens Ment Health 2008; 11:57-65.

28. Milgrom J, Gemmill AW, Bilszta JL, et al. Antenatal risk factors for postnatal depression: a large prospective study. J Affect Disord 2008;108:147-57.

29. Laughlin L. Maternity leave and employment patterns: 2006-2008. Current Population Report. Washington, DC: U.S. Census Bureau; 2011: P70-128.

30. Dagher RK, McGovern PM, Alexander BH, Dowd BE, Ukestad LK, McCaffrey DJ. The psychosocial work environment and maternal postpartum depression. Int J Behav Med 2009;16:339-46.

31. Grice MM, McGovern PM, Alexander BH, Ukestad LU, Hellerstedt $W$. Balancing work and family after childbirth: a longitudinal study. Womens Health Issues 2011;21:19-27.

32. O'Hara MW. The nature of postpartum depressive disorders. In: Murray L, Cooper PJ, editors. Postpartum Depression and Child Development. New York: Guilford Publications; 1997: 3-31.

33. Gjerdingen DK, Froberg DG, Chaloner KM, McGovern PM. Changes in women's physical health during the first postpartum year. Arch Fam Med 1993;2:277-83.

34. Kroenke K, Spitzer RL, Williams JBW. The Patient Health Questionnaire-2: validity of a two-item depression screener. Med Care 2003;41:1284-92.

35. Declercq ER, Sakala C, Corry MP, Applebaum S. Listening to Mothers II: Report of the Second National Survey of Women's Childbearing Experiences. New York: Childbirth Connection; 2006.

36. Rubertsson C, Wickberg B, Gustavsson P, Radestad I. Depressive symptoms in early pregnancy, two months and one year postpartum-prevalence and psychosocial risk factors in a national Swedish sample. Arch Womens Ment Health 2005;8:97-104.

37. Berenson AB, Breitkopf CR, Wu ZH. Reproductive correlates of depressive symptoms among low-income minority women. Obstet Gynecol 2003;102: 1310-7.

38. Miyake Y, Tanaka K, Sasaki S, Hirota Y. Employment, income, and education and risk of postpartum depression: the Osaka Maternal and Child Health Study. J Affect Disord 2011;130(1-2):133-7.

39. Christ SL, Lee DJ, Fleming LE, et al. Employment and occupation effects on depressive symptoms in older Americans: does working past age 65 protect against depression? J Gerontol B Psychol Sci Soc Sci 2007;62:S399-403.

40. Jahoda M. Work, employment, and unemployment: values, theories, and approaches in social research. Am Psychol 1981;36:184-91. 